\title{
Multilayer Thin Films as Pseudo-Homogeneous EDX Standards
}

\author{
Hendrik O. Colijn ${ }^{1}$, Jonathan Orsborn ${ }^{1}$, Daniel Chmielewski ${ }^{2}$, and David W. McComb ${ }^{1}$. \\ 1. Ohio State University, Center for Electron Microscopy \& Analysis, Columbus, OH, USA. \\ 2. Ohio State University, Electrical \& Computer Engineering, Columbus, OH, USA.
}

Quantifying EDX data in the TEM/STEM is generally accomplished using either the Zeta-factor or the Cliff-Lorimer method (k-factors) [1]. The Zeta-factor method requires accurate measurement of the beam current which can be difficult to measure accurately on most microscopes. Consequently the Zeta-factor approach is not frequently used. The Cliff-Lorimer method is much more commonly used but requires accurate $\mathrm{k}$-factors in the analysis. While theoretical k-factors are available there can easily be large variations in the values of the k-factor depending on the theoretical model chosen. These variations can lead to errors in the final composition values. Experimentally determined k-factors are generally more reliable for doing the analysis but require the availability of suitable experimental standards.

Suitable standards can be difficult to obtain and issues such as inhomogeneities and surface films due to oxidation etc. can complicate the analyses. Advances in thin film growth techniques now allow the deposition of nanometer scale uniform thin films of known composition. High quality thin films can be grown by a number of techniques including Atomic Layer Deposition (ALD), Radio Frequency (RF) sputtering, and Molecular Beam Epitaxy (MBE). If the sample has sufficient layers, one can achieve a reasonable approximation of a homogeneous material. This does require that the exact thickness and composition of each layer be well known. Cross-section imaging of the multi-layers allows accurate thickness measurements to be made. In many cases, one can very accurately determine the layer thicknesses by counting the number of atomic planes.

We have used multi-layer standards to measure k-factors for several elemental systems. We verified the layer thicknesses by preparing cross-sections using a FIB with subsequent TEM analysis. Multiple spectra were then taken at various thicknesses of the material. We employed several software packages such as FEI TIA and EDAX TEAM to determine the peak intensities. The intensity ratios from both packages were similar. A regression fit on these intensity ratios was used to extrapolate them to zero thickness using the van Capellen method [2]. The regression fit also provides an estimate of the uncertainly in the $\mathrm{y}$-intercept value which gives an estimate of the bias error in the Cliff-Lorimer quantification method.

Figure 1 shows a STEM image of an MBE grown multilayer of AlGaAs and InGaP used in this experiment. The layer thicknesses were confirmed by HRTEM. Figure 2 shows the intensity ratios for several elements that were in the multilayer film and the regression fit to find the zero-thickness intensity ratio. Using the layer thicknesses and the layer composition we calculated the average composition of the multilayer. The kab values were then determined by using the average composition and the y-intercept of the intensity ratio. [3]

\section{References}

[1] see e.g. D.B. Williams \& C.B. Carter, Transmission Electron Microscopy: A Textbook for Materials Science, (Springer), 2009, chapter 35.

[2] E. van Capellen, Microsc. Microanal. Microstruct. 1, (1990), pp. 1-22. 
[3] The authors acknowledge support from The Ohio State University and the Ohio Third Frontier Research Scholar program.
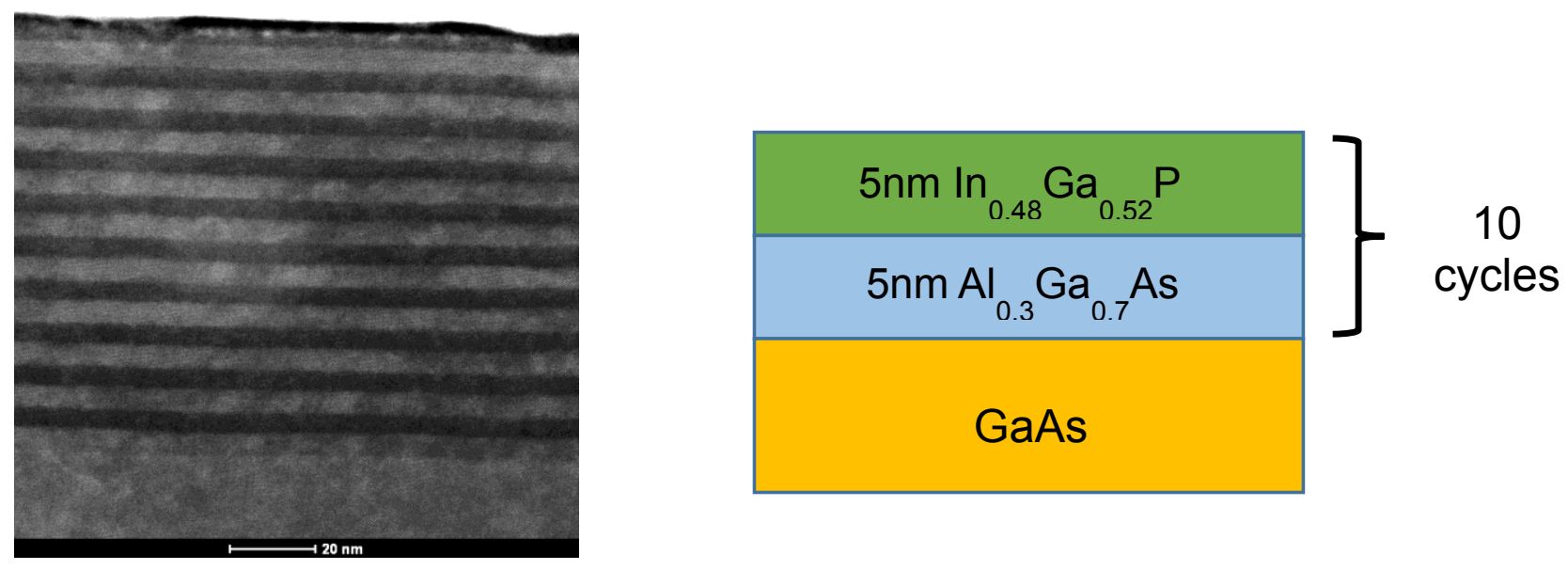

Figure 1. HAADF STEM image of MBE grown multilayer film and schematic of the layers.
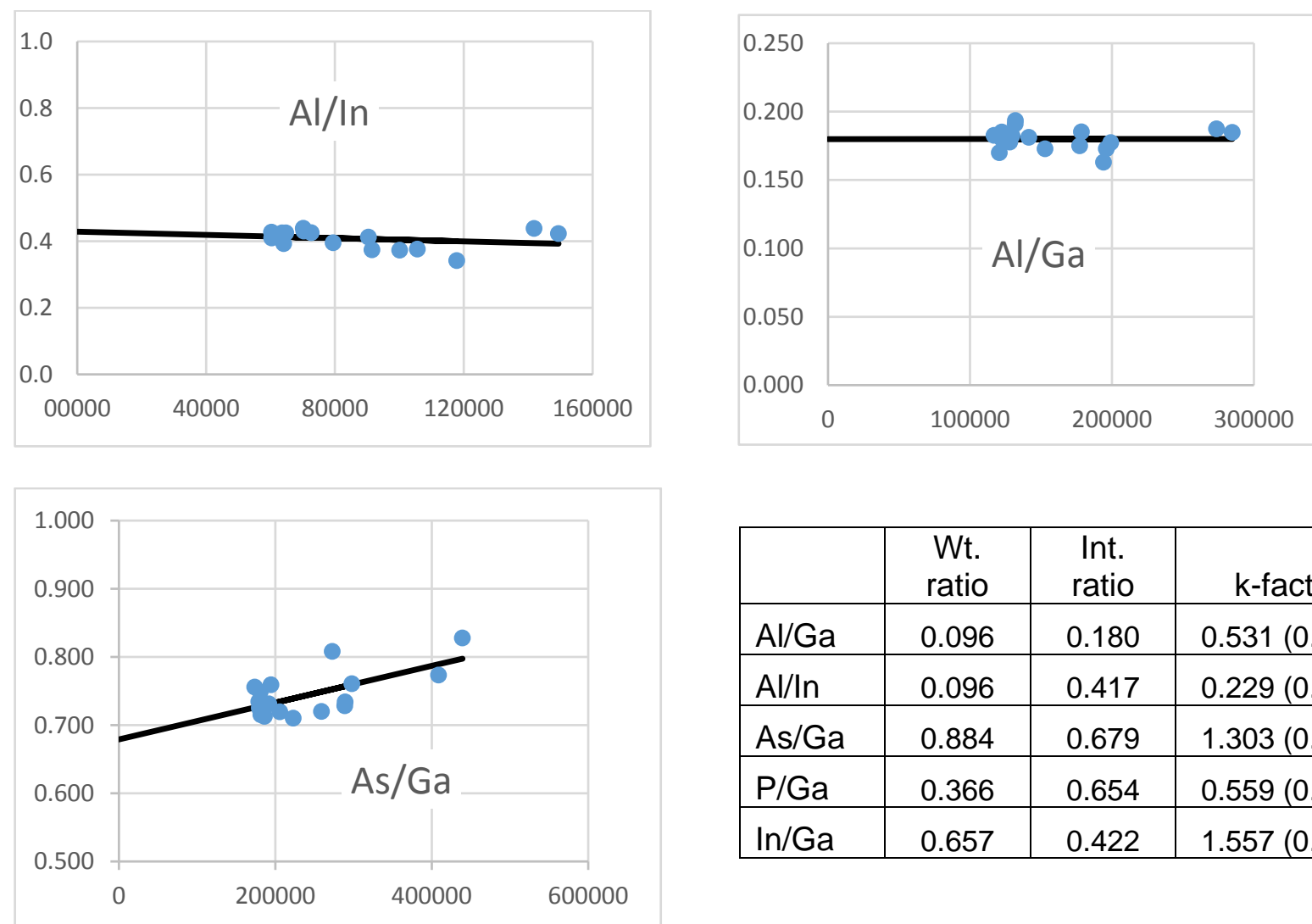

\begin{tabular}{|l|c|c|c|}
\hline & $\begin{array}{c}\text { Wt. } \\
\text { ratio }\end{array}$ & $\begin{array}{c}\text { Int. } \\
\text { ratio }\end{array}$ & k-factor \\
\hline $\mathrm{Al} / \mathrm{Ga}$ & 0.096 & 0.180 & $0.531(0.019)$ \\
\hline $\mathrm{Al} / \mathrm{In}$ & 0.096 & 0.417 & $0.229(0.013)$ \\
\hline $\mathrm{As} / \mathrm{Ga}$ & 0.884 & 0.679 & $1.303(0.037)$ \\
\hline $\mathrm{P} / \mathrm{Ga}$ & 0.366 & 0.654 & $0.559(0.025)$ \\
\hline $\mathrm{In} / \mathrm{Ga}$ & 0.657 & 0.422 & $1.557(0.070)$ \\
\hline
\end{tabular}

Figure 2. Typical intensity ratio plots with regression fit and calculated zero thickness k-factors with the $1 \sigma$ uncertainty in parentheses. 